\title{
A cost-effectiveness evaluation comparing originator follitropin alfa to the biosimilar for the treatment of infertility
}

This article was published in the following Dove Press journal:

International Journal of Women's Health

7 December 2016

Number of times this article has been viewed

\author{
Salvatore Gizzo' \\ Juan A Garcia-Velasco² \\ Franca Heiman ${ }^{3}$ \\ Claudio Ripellino ${ }^{3}$ \\ Klaus Bühler ${ }^{4}$ \\ 'Department of Woman and Child \\ Health, University of Padua, Padua, \\ Italy; ${ }^{2}$ Department of Obstetrics \\ and Gynecology, Rey Juan Carlos \\ University Madrid, Madrid, Spain; ${ }^{3}$ MS \\ Health Information Solutions Italy Srl, \\ Milan, Italy; ${ }^{4}$ Centre for Gynaecology, \\ Endocrinology, and Reproductive \\ Medicine, Ulm and Stuttgart, Germany
}

Correspondence: Claudio Ripellino IMS Health Information Solutions Italy Srl, Via Fabio Filzi 29, Milan 20124, Italy

Tel +390269786291

Fax +390270039919

Email cripellino@it.imshealth.com
Objectives: To perform a cost-effectiveness evaluation comparing the originator follitropin alfa $\left(\right.$ Gonal- $\left.^{\circledR}\right)$ to the biosimilar $\left(\right.$ Bemfola $\left.^{\circledR}\right)$ in the Italian and Spanish contexts, with an assessment of the German and UK backgrounds.

Methods: Starting from the study by Rettenbacher et al, a cost-effectiveness model was developed in the Italian and Spanish contexts. Clinical data on subjects, doses of gonadotropin, pregnancies, live-born children, and ovarian hyperstimulation syndrome were used to feed the model. Costs related to drugs, hospitalizations, specialist visits, and examinations were retrieved from Italian and Spanish tariffs. Gonadotropin acquisition costs for Germany and the UK were also taken into account to expand the economical assessment to the other countries. The evaluation was done based on the National Health Service perspective. Sensitivity analyses, both univariate and probabilistic, as long as scenario analyses, tested the robustness of the model.

Results: Originator follicle-stimulating hormone (FSH) costs were $€ 3,663$ and $€ 6,387$ in Italy and Spain, respectively, whereas biosimilar FSH costs were $€ 3,483$ and $€ 6,342$. The efficacy was found to be 0.52 for the originator and 0.47 for the biosimilar. The average cost per live birth was estimated to be $€ 7,044$ and $€ 12,283$ for the originator FSH and $€ 7,411$ and $€ 13,494$ for the biosimilar for Italy and Spain, respectively. Furthermore, the originator FSH generated an incremental cost-effectiveness ratio of $€ 3,600$ for Italy and $€ 900$ for Spain compared to the biosimilar. Sensitivity analyses confirmed the results of the base case model.

Conclusion: This analysis indicated that the originator FSH is a cost-efficient treatment strategy for Italian and Spanish health services compared to the biosimilar and it would be worthwhile extending this evaluation to other countries.

Keywords: FSH, follitropin alfa, biosimilar, infertility

\section{Introduction}

After patent expiry of the originator, biosimilar products, that is, biological medicines made by or derived from a biological source, such as bacteria or yeast, may be developed, thus offering the possibility of greater access to effective and safe treatment options at a more affordable price than the originator biologics. Biosimilar medicines are approved based on demonstration that they are highly similar to the approved biological products meeting high standards for comparability to the originator medicine and for use in the same indications. ${ }^{1}$ Only minor differences in clinically inactive components are allowed in biosimilar products. ${ }^{2}$

The biosimilar follitropin alfa (Bemfola ${ }^{\circledR}$, Finox AG, Burgdorf, Switzerland) is a biological medicinal product that is similar to the originator (Gonal- $\mathrm{f}^{\mathbb{B}}$, Merck Serono, Darmstadt, Germany). It is the first recombinant follicle-stimulating hormone (FSH) 
biosimilar launched in the European market and it was approved by the European Medicines Agency on March 27, 2014. ${ }^{3}$ The biosimilar FSH has nonclinical pharmacological, pharmacokinetic, and toxicological profiles, which are similar to those of the originator FSH and it is licensed for all indications of the reference product. ${ }^{4,5}$

Clinical efficacy was demonstrated in an assessor-blinded, randomized, parallel-group, multicenter, phase III study conducted in women undergoing assisted reproduction techniques at 15 centers in six European countries. ${ }^{6}$ Clinical equivalence of biosimilar follitropin alfa to the originator product was demonstrated in terms of the number of oocytes retrieved during stimulation of multifollicular development in patients undergoing superovulation for assisted reproductive technology (ART) with a between-treatment group difference of 0.27 (95\% CI: -1.34 to 1.32). Furthermore, clinical and ongoing pregnancy and live-born children were higher in the originator FSH group for treatment cycle 1 while for treatment cycle 2 were higher in the biosimilar group, either way not significant. Ovarian hyperstimulation syndrome (OHSS) is one of the most important adverse events of gonadotropin and, in the reference study, more patients in the biosimilar group in comparison to the originator group, $5.6 \%$ vs $3.3 \%$, reported it.

Currently, whether the lower cost of the biosimilar follitropin alfa does provide an improvement in cost-effectiveness has not yet been demonstrated. The aim of this study was indeed to perform a cost-effectiveness analysis (CEA) comparing the cost and efficacy of the two comparators (originator follitropin alfa vs biosimilar) in the Italian and Spanish contexts, with an assessment of the German and UK backgrounds.

\section{Materials and methods}

The analysis was based on previously conducted studies and did not involve any new studies with human or animal subjects performed by any of the authors. The study was performed in accordance with ISPOR Good Practices for Outcomes Research and relevant international and national guidelines for health economics studies. Ethics board approval and informed consent were not required because the project did not involve human or animal participants and the analysis only used publicly available anonymized data.

\section{Clinical evidence}

The clinical evidence on which the economic analysis was based was retrieved from a single study conducted by Rettenbacher et $\mathrm{al}^{6}$ as other publications comparing the biosimilar to the originator follitropin alfa are not available. This multicenter phase III study compared the efficacy and safety of the originator to the biosimilar in 333 women undergoing ovarian stimulation for in vitro fertilization (IVF) for ART superovulation. Most patients $(>75 \%)$ were younger than 35 years of age and women enrolled have undergone both intracytoplasmic sperm injection (ICSI) and IVF according to the center's standard procedures. In particular, the ICSI method was more applied for the oocytes retrieved in the Gonal-f group (59.3\% vs 55.31\%), whereas the IVF method was more applied in the Bemfola group (44.7\% vs $40.7 \%$ ). Clinical data on the number of subjects, the total dose of gonadotropin, pregnancies and live-born children, and OHSS for both first and second cycle were used to feed the model (Table 1).

In the first cycle of treatment, 220 patients in the biosimilar FSH group and 113 patients in the originator group were assessed. The mean total dose for r-hFSH was 1555.7 $( \pm 293.0)$ IU for the biosimilar, while it was $1569.2( \pm 259.20)$ IU for the originator. At cycle 1, the clinical pregnancy rate per embryo transfer was $40.2 \%$ for the biosimilar and $48.2 \%$ for the originator, whereas patients with live-born children were $80(35.7 \%)$ and $50(43.9 \%)$, respectively. At the second treatment cycle, 72 patients in the biosimilar group and 38 in the originator group were treated with a mean total $\mathrm{r}-\mathrm{hFSH}$ dose of $1612.3( \pm 217.7)$ IU for the biosimilar follitropin alfa and $1604.9( \pm 216.61)$ IU for the originator. The clinical pregnancy rate at cycle 2 was $38.5 \%$ for the biosimilar FSH and $27.8 \%$ for the originator, whereas the ongoing pregnancy

Table I Model input data: clinical background

\begin{tabular}{|c|c|c|}
\hline Clinical data & $\begin{array}{l}\text { Biosimilar } \\
\text { FSH }\end{array}$ & $\begin{array}{l}\text { Originator } \\
\text { FSH }\end{array}$ \\
\hline \multicolumn{3}{|l|}{ Treatment cycle I } \\
\hline Number of subjects & 220 & 113 \\
\hline Mean (SD) total dose r-FSH (IU) & I,555.7 (293.00) & I,569.2 (259.20) \\
\hline $\begin{array}{l}\text { Clinical pregnancy rate per } \\
\text { embryo transfer (\%) }\end{array}$ & $90(40.2)^{\mathrm{a}}$ & $55(48.2)^{\mathrm{a}}$ \\
\hline Patients with live-born children (\%) & $80(35.7)^{\mathrm{a}}$ & $50(43.9)^{\mathrm{a}}$ \\
\hline \multicolumn{3}{|l|}{ Treatment cycle 2} \\
\hline Number of subjects & 72 & 38 \\
\hline Mean (SD) total dose r-FSH (IU) & $1,612.3(2 \mid 7.67)$ & $1,604.9(216.6 I)$ \\
\hline $\begin{array}{l}\text { Clinical pregnancy rate per } \\
\text { embryo transfer (\%) }\end{array}$ & $25(38.5)^{b}$ & $10(27.8)^{\mathrm{b}}$ \\
\hline Patients with live-born children (\%) & $22(33.8)^{b}$ & $9(25)^{b}$ \\
\hline \multicolumn{3}{|l|}{ Treatment cycle I } \\
\hline OHSS (\%) & $14(5.6)^{d}$ & $4(3.3)^{c}$ \\
\hline \multicolumn{3}{|l|}{ Grades of OHSS } \\
\hline Mild/moderate (\%) & 4.8 & 2.5 \\
\hline Severe (\%) & 0.8 & 0.8 \\
\hline
\end{tabular}

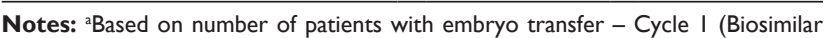
FSH: 224; Originator FSH: I I4); 'based on number of patients with embryo transfer Cycle 2 (Biosimilar FSH: 65; Originator FSH: 36); 'OHSS; dbased on per-protocol population (Biosimilar FSH: 249; Originator FSH: 123). Data derived from European medicines agency assessment report. ${ }^{3}$

Abbreviations: $\mathrm{FSH}$, follicle-stimulating hormone; OHSS, ovarian hyperstimulation syndrome; SD, standard deviation. 
rate was $33.8 \%$ and $25 \%$, respectively. OHSS was assessed only for the first treatment cycle and occurred in $14(5.6 \%)$ of the biosimilar cohort vs $4(3.3 \%)$ of the originator cohort. The majority of the latter $(4.8 \%$ and $2.5 \%$ for the biosimilar and the originator, respectively) were mild-to-moderate.

\section{Costs}

The economic evaluation has been performed from the Italian and Spanish National Health Services (NHS) perspective. Table 2 reports all costs taken into account, which are expressed in Euros. This analysis considered the diagnosisrelated group (DRG) tariff 359, which covers all ART procedures, excluding gonadotropin drugs, follow-up visits, and adverse events costs that have been quoted separately. The tariff was retrieved from the "Tariffa Unica Convenzionale" (TUC) 2013 pricelist from the Italian Ministry of Health website and from "Registro de Altas de los Hospitales Generales del Sistema Nacional de Salud" for the Italian and Spanish contexts, respectively. ${ }^{7,8}$ In Italy, unit costs of $€ 27.38$ for the originator FSH and $€ 21.90$ for the biosimilar FSH per 75 IU vial of r-hFSH activity were derived from "the Official Italian Bulletin", whereas in Spain both have the same unit

Table 2 Model input data: health costs

\begin{tabular}{|c|c|c|}
\hline Cost data & $\begin{array}{l}\text { Cost per unit } \\
\text { in } €-\text { Italy }\end{array}$ & $\begin{array}{l}\text { Cost per unit } \\
\text { in } €-\text { Spain } \\
\end{array}$ \\
\hline \multicolumn{3}{|l|}{ Routine intervention } \\
\hline $\begin{array}{l}\text { DRG } 359 \text { "uterus and adnexa } \\
\text { nonmalignant, without cost } \\
\text { specification" }\end{array}$ & $2,124.52^{7}$ & $4,289.51^{8}$ \\
\hline $\begin{array}{l}\text { Ex-factory price Originator FSH } \\
\text { (75 IU) }\end{array}$ & $27.38^{9}$ & $20.16^{10}$ \\
\hline $\begin{array}{l}\text { Ex-factory price Biosimilar FSH } \\
\text { (75 IU) }\end{array}$ & $21.90^{9}$ & $20.16^{10}$ \\
\hline $\begin{array}{l}\text { Follow-up: blood test for beta- } \\
\text { HCG (pregnancy test) }\end{array}$ & $9.98^{7}$ & $15.00^{10}$ \\
\hline $\begin{array}{l}\text { Clinical follow-up visits until first } \\
\text { ultrasound: gynecological visit + } \\
\text { ultrasound of lower abdomen }\end{array}$ & $52.68^{7}$ & $83.07^{10}$ \\
\hline \multicolumn{3}{|l|}{ OHSS ${ }^{\text {a }}$ mild/moderate } \\
\hline N.2 gynecological visit & $20.66^{7}$ & $50.90^{10}$ \\
\hline N.2 ultrasound of lower abdomen & $32.02^{7}$ & $32.17^{10}$ \\
\hline N.2 hematology laboratory tests & $7.4 I^{7}$ & $10.60^{10}$ \\
\hline N.2 hematocrit laboratory tests & $0.69^{7}$ & $6.11^{10}$ \\
\hline N.2 creatinine laboratory test & $1.13^{7}$ & $2.90^{10}$ \\
\hline N.2 electrolytes laboratory tests & $4.30^{7}$ & $3.86^{10}$ \\
\hline N.2 hepatic laboratory tests & $14.30^{7}$ & $14.50^{10}$ \\
\hline \multicolumn{3}{|l|}{ OHSS severe } \\
\hline $\begin{array}{l}\text { DRG } 369 \text { "Menstrual disorders } \\
\text { and other disorders of the female } \\
\text { reproductive system" }\end{array}$ & $800.35^{7}$ & $\mathrm{I}, 657.28^{8}$ \\
\hline
\end{tabular}

Note: ${ }^{\mathrm{O}} \mathrm{OHSS}$.

Abbreviations: DRG, diagnosis-related group; FSH, follicle-stimulating hormone; OHSS, ovarian hyperstimulation syndrome. costs, which is $€ 20.16$ per 75 IU vial. ${ }^{9,10}$ Data on follow-up visits, that is, blood test for beta-HCG and clinical follow-up visits until the first ultrasound, were retrieved from TUC 2013 pricelists for Italy and from the eSalud Sanitary costs database for Spain. ${ }^{7,10}$ Regarding OHSS, two different tariffs were applied based on OHSS severity: for mild/moderate OHSS, the tariff included gynecological visits and laboratory tests for an amount of $\sim € 160$ and $€ 120$ for Italy and Spain, respectively; severe OHSS tariff entailed a hospitalization (DRG 369) with an amount of $~ € 800$ and $€ 1,600$ for Italy and Spain, respectively. Furthermore, mild/moderate OHSS patients were also treated with paracetamol and metoclopramide, but since these drugs are dispensed as class $\mathrm{C}$ in Italy, that is, are fully charged to the patient, related costs were not included in the Italian model, whereas in the Spanish model they were accounted for (€1.14) considering that the Spanish NHS reimburses $60 \%$ of the cost for the majority of prescription-only pharmaceuticals. Gonadotropin acquisition costs for Germany and the UK were also taken into account to expand the economical assessment to the other countries.

\section{Model design}

CEA is a method of assessing health gains in relation to the costs of different health interventions. A decision tree model was developed using TreeAge Pro ${ }^{\mathrm{TM}}$ software 2015 and all costs described in the costs section were applied to the clinical data retrieved from the study by Rettenbacher et al, ${ }^{6}$ simulating both Italian and Spanish contexts. At the first cycle of treatment, the DRG 359 cost together with gonadotropin costs, calculated as unitary $\mathrm{r}-\mathrm{hFSH}$ costs multiplied by mean total dose, was applied to each patient entering in the model for each treatment arm (biosimilar and originator). Subsequently, the cost of blood test for beta-HCG to assess pregnancy was considered and clinical follow-up visits costs were applied to women who got pregnant according to the study subjects for each arm. For the first cycle of treatment, OHSS costs were estimated for each arm according to the probability of experiencing OHSS and the unitary OHSS costs mentioned earlier. The same process was performed to retrieve total costs associated to the second cycle of treatment according to the number of women, the mean total dose of gonadotropin and pregnancy rates. Total costs of biosimilar and originator FSH arms for Italy and Spain were obtained by summing up all costs derived from both the first and second cycles of treatment and were then divided by the efficacy parameter, represented by live birth rates, in order to obtain average costs per live birth for the two countries. The incremental cost-effectiveness ratio (ICER) was calculated as the 
difference in costs divided by the difference in efficacy of the two treatment arms for both Italy and Spain. Univariate sensitivity analyses considering efficacies, gonadotropin costs, OHSS rates, and mean dosages were performed to test the robustness of the results. Furthermore, a scenario analysis considering second cycle OHSS rates as the first cycle ones was performed. Uncertainty in the model was assessed using probabilistic sensitivity analysis, where the value for each parameter was determined independently from a probability distribution (gamma distributions for costs and beta-distributions for probabilities), and the results were recalculated for each of the 10,000 simulations performed.

\section{Results}

The results of the Italian and Spanish CEA are shown in Table 3. Total costs obtained were $€ 3,483$ and $€ 6,342$ for the biosimilar FSH and $€ 3,663$ and $€ 6,387$ for the originator in Italy and Spain, respectively. The efficacy, that is, live-birth rate, for the originator FSH was 0.52 , whereas it was 0.47 for the biosimilar. In other words, there will be 52 live-born children per 100 patients treated with the originator and 47 live-born children per 100 patients treated with the biosimilar. The average cost per live-birth was estimated to be $€ 7,044$ and $€ 12,283$ for the originator and $€ 7,411$ and $€ 13,494$ for the biosimilar for Italy and Spain, respectively, underlying the lower cost per live-birth for the originator follitropin alfa. Furthermore, the originator FSH generated an ICER of $€ 3,600$ for Italy and $€ 900$ for Spain compared to the biosimilar FSH, which is the additional cost required for the originator to gain an additional live-birth in comparison with the biosimilar. The originator, although it has a higher acquisition cost in Italy and the same acquisition cost in Spain, provides a lower average cost per live-birth and an ICER of $€ 3,600$ and $€ 900$ for Italy and Spain, respectively.

Figures 1 and 2 describe Tornado diagram for Italy and Spain, respectively, and provide a way for clearly identifying the factors whose uncertainty drives the largest impact on ICERs. These figures indicate that uncertainty in efficacies has a bigger impact on ICER values in comparison with the other parameters. In fact, by varying efficacies from the lower to the upper confidence interval, ICERs varied from $€ 1,877$ to $€ 7,642$ for Italy and from $€ 460$ to $€ 1,871$ for Spain.

Results from the scenario analyses showed no relevant differences in ICERs from the base case (Italy: $€ 3,600$; Spain: €900). Figures 3 and 4 show the cost-effectiveness acceptability curves for the probability that the originator is cost-effective compared to the biosimilar, for a range that the NHS might consider as the maximum cost it is willing to pay for a gain in one live-born child. In Italy, if the NHS willingness to pay threshold was greater than $€ 3,600$, then the originator would have a higher probability to be costeffective compared to the biosimilar. Below this threshold, the biosimilar would have a higher probability to be cost effective. In Spain, the NHS willingness to pay threshold beyond which the originator would have a higher probability to be cost effective compared to the biosimilar was $€ 900$.

Similar to Spain, in the UK, the originator and biosimilar FSH have the same acquisition cost (£23.50), whereas in Germany the originator and biosimilar acquisition costs are $€ 53.69$ and $€ 42.95$, respectively, generating a delta acquisition cost quite high in comparison to Italy (€10.74 for Germany vs $€ 5.48$ for Italy).

\section{Discussion}

The biosimilar medicines market uptake in Europe is moderate, with variations by country: in volume it is $\sim 20 \%-30 \%$ in Spain, the UK, and France, remaining significantly behind Germany, which has $\sim 50 \%$ volume uptake. ${ }^{11}$ In Italy, the uptake of biosimilar medicines in 2013 was $€ 3.7$ billion, accounting for $\sim 13 \%$ of the overall expenditure and for $16.4 \%$ of the expenditure for NHS-reimbursed medicines and 34.5\% for the Health Services volume of medicines. In Italy, the biosimilar policy includes a mandatory discount of 15\%-22\% vs the branded product. ${ }^{12}$ Standard literature searches conducted by the authors did not identify any published costeffectiveness analyses of the biosimilar gonadotropin, with

Table 3 Results of the CEA ${ }^{a}$

\begin{tabular}{|c|c|c|c|c|c|c|}
\hline Strategy & Cost $(€)$ & Incremental cost $(€)$ & Efficacy & Incremental efficacy & $\operatorname{ICER}^{\mathrm{b}}(€)$ & Cost per live birth $(€)$ \\
\hline \multicolumn{7}{|l|}{ Italy } \\
\hline Biosimilar FSH & 3,483 & - & 0.47 & - & - & $7,4 I I$ \\
\hline Originator FSH & 3,663 & 180 & 0.52 & 0.05 & 3,600 & 7,044 \\
\hline \multicolumn{7}{|l|}{ Spain } \\
\hline Biosimilar FSH & 6,342 & & 0.47 & & & 13,494 \\
\hline Originator FSH & 6,387 & 45 & 0.52 & 0.05 & 900 & 12,283 \\
\hline
\end{tabular}

Notes: aCEA; '।CER.

Abbreviations: CEA, cost-effectiveness analysis; FSH, follicle-stimulating hormone; ICER, incremental cost-effectiveness ratio. 


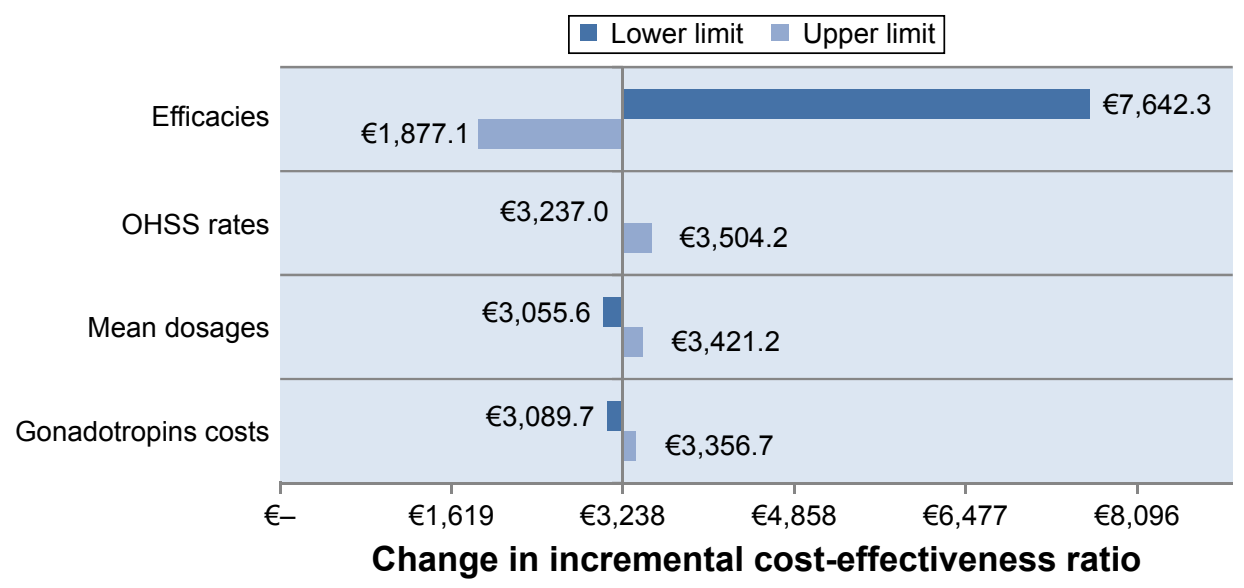

Figure I Tornado diagram - Italy.

Abbreviation: OHSS, ovarian hyperstimulation syndrome.

cost-minimization analyses only available. ${ }^{13,14}$ However, this kind of analysis defines the cost per course of treatment of biosimilar FSH with respect to the originator on the base of equivalent therapeutic equivalence (including adverse reactions, complications, and duration of therapy). The All Wales Strategy Medicines Group and the Scottish Medicines Consortium reviews of comparative health economic evidence were based on drug costs only and concluded that the net medicines budget impact for the biosimilar as compared with the originator was estimated to be cost-neutral in both the lowest dose and highest dose scenarios. ${ }^{13,14}$ However, costs were based on assumption of the prefilled formulations used in practice in Wales and Scotland. In addition, as with other biosimilar medicines, there is a lack of clinical data comparing the biosimilar FSH with the reference product across the range of indications; therefore, the conclusion of equivalence is assumed to extrapolate to the other indications covered by the reference product. The comparative health economic evidence and the relevant cost-minimization approach assume equivalence in all domains of health outcomes.

This study is the first CEA comparing the originator recombinant gonadotropin and the biosimilar FSH in women undergoing ovarian stimulation for IVF for ART superovulation in Italy and Spain. It was found that the average cost per live-birth is lower for treatment with the originator $r$-hFSH than for treatment with the biosimilar r-hFSH. The ICER value for live-birth for the originator compared to the biosimilar was estimated to be $€ 3,600$ and $€ 900$ in Italy and Spain, respectively. However, from a cost-effectiveness point of view, the preferred strategy depends on the NHS willingness to pay: if the NHS is willing to pay less than the value of the ICER for one extra live-born child, the biosimilar FSH will be preferred;

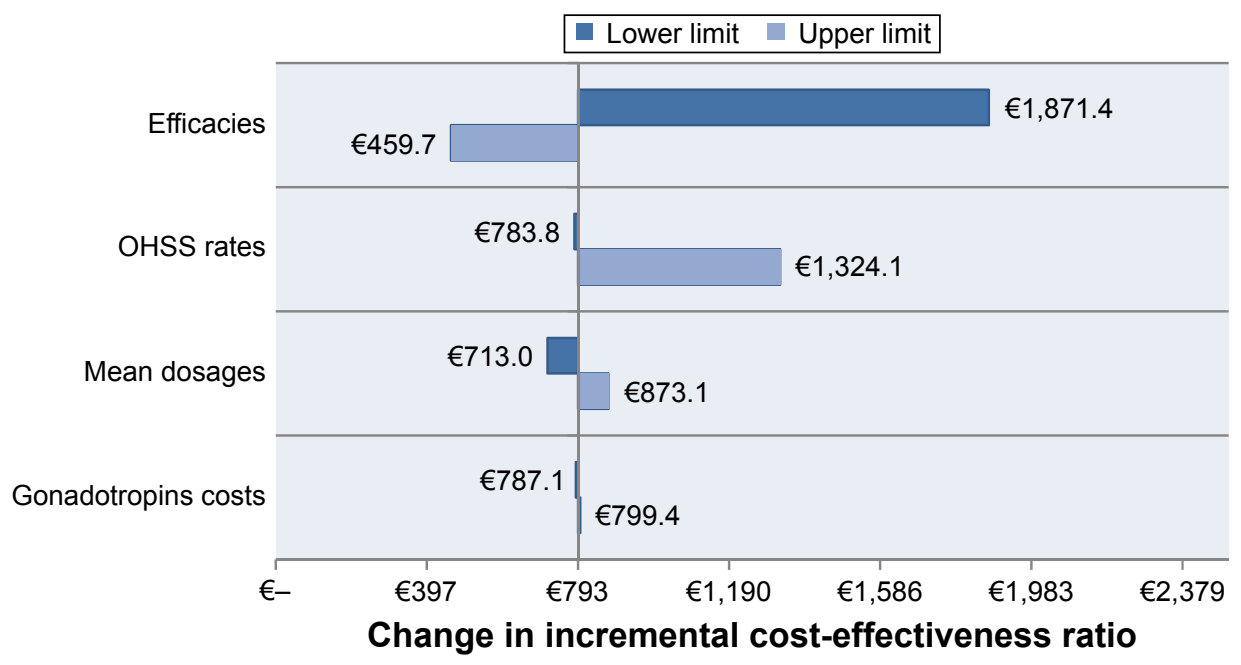

Figure 2 Tornado diagram - Spain.

Abbreviation: OHSS, ovarian hyperstimulation syndrome. 


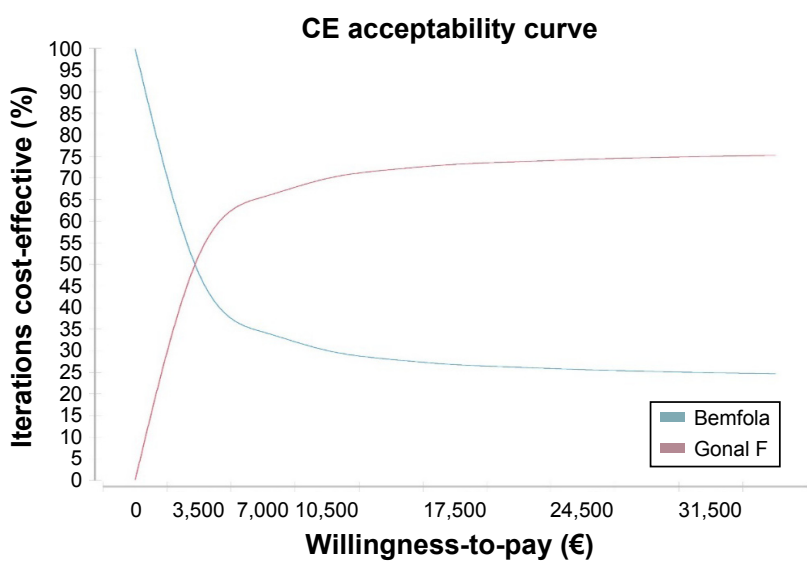

Figure 3 CE acceptability curve - Italy. Abbreviation: $C E$, cost effectiveness.

if it is willing to pay the value of the ICER or more per extra live-born child, the originator FSH will be preferred. Since no national or international thresholds have been defined regarding ICER per live-birth, no clear indications on the willingness to pay per live-birth can be supported by the authors. Sensitivity analyses confirmed the results from the base case scenario showing no important differences in ICERs by varying key parameters and confirming the robustness of the model.

In the UK, given the same acquisition costs for the originator and biosimilar FSH, authors could predict potential preferable cost-effectiveness performances for the originator follitropin alfa in comparison to the biosimilar due to a lower gonadotropin cost impact, as for Spain, whereas the German cost-effectiveness should be assessed in depth. Nevertheless, health systems are different among countries and it would be worthwhile examining in depth the UK and German costeffectiveness considering not only gonadotropin acquisition costs but also reimbursement systems, interventions, and procedures related to ART.

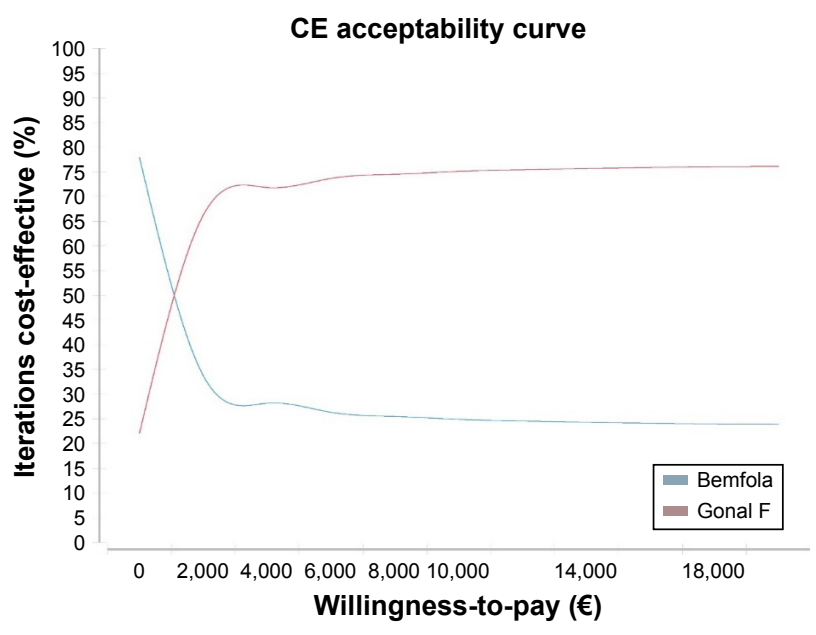

Figure 4 CE acceptability curve - Spain.
Pharmacoeconomic studies fed with data reported in the literature (such as the one here presented) suffer from biases and limitations and cannot substitute direct real-life comparisons. The study by Rettenbacher et $\mathrm{al}^{6}$ was not powered to demonstrate the live-birth rate and it may be an important limitation of the present study. Furthermore, since the objective of the second treatment cycle was to assess the immunogenicity and safety of the biosimilar FSH, data on second cycle efficacy considered in this model could be biased both for the clinical design of the study and for the small number of patients who underwent the second treatment cycle. Indeed, if the authors had considered for the Spanish scenario a real-life approach where the chance of a second cycle results from the success rate in the previous one for the originator from Rettenbacher et al, ${ }^{6}$ the results of the model would have presented the originator FSH as both more effective in comparison with the biosimilar $(0.58$ vs 0.57 , respectively) and less costly (€7,455 vs $€ 7,848$, respectively). Thus, in the Spanish scenario, this real-life approach would yield that the originator FSH is a dominant strategy when compared to the biosimilar. Another potential limitation is that the biosimilar follitropin alfa is available only as single-use, fixed-dose, prefilled pens in contrast to the originator, which is available as multidose vials and prefilled pens. As doses need to be individually tailored to response, it is not possible to determine the impact of potential dose wastage on costs arising from the use of the different presentations. In addition, in Rettenbacher et al, ${ }^{6}$ there was a higher proportion of patients treated with the biosimilar FSH and anti-Müllerian hormone levels ( $\geq 24 \mathrm{pmol} / \mathrm{L}$ ) at baseline, which could have contributed to the higher OHSS incidence in this treatment group. ${ }^{15,16}$ Furthermore, dissimilarities in dose reduction were observed between the biosimilar and originator groups, which also could have resulted in a higher incidence of OHSS for the biosimilar follitropin alfa. ${ }^{4}$ Clinical outcomes data stratified by age groups and types of ART would have access to model sub-populations avoiding biases on treatment-related benefits and potential harms due to different population characteristics. Despite these limitations, results of this CEA indicate that the originator FSH is a cost-efficient treatment strategy for the Italian and Spanish health services compared to the biosimilar in the treatment of infertility and require further investigation for the other countries. The reliability of cost-effectiveness researches can be greatly improved over time as evidence continues to grow and long-term data are generated correlating to efficacy in the individual patient populations of interest. 


\section{Acknowledgments}

This study was financially supported by Merck Serono Spa, which was not responsible for the creation of study documents, data analysis, data interpretation, or writing of the manuscript.

\section{Author contributions}

$\mathrm{CR}$ and $\mathrm{FH}$ were responsible for the creation of study documents, data analysis, data interpretation, and writing of the manuscript. All authors contributed toward data analysis, drafting and revising the paper and agree to be accountable for all aspects of the work.

\section{Disclosure}

$\mathrm{CR}$ and FH are employees of IMS Health Information Solutions Italy Srl, and SG and KB have received honoraria from Merck Serono Spa. The other authors report no conflicts of interest in this work.

\section{References}

1. Weise M, Bielsky MC, De Smet K, et al. Biosimilars: what clinicians should know. Blood. 2012;120(26):5111-5117.

2. FDA. Available from: http://www.fda.gov/drugs/developmentapprovalprocess/howdrugsaredevelopedandapproved/approvalapplications/therapeuticbiologicapplications/biosimilars/default.htm. Accessed March 1, 2016.

3. European Medicines Agency, Committee for Medicinal Products for Human Use (CHMP). Bemfola Assessment Report. 2014.

4. European Medicines Agency. Assessment Report for Bemfola (R) Procedure No:EMEA/H/C/002615. 2014.
5. Sanghvi S. Assessment of bemfola ${ }^{\circledR}$ : biosimilar follitropin alfa. J Form Med Man. 2015;1:4-6.

6. Rettenbacher M, Andersen AN, Garcia-Velasco JA, et al. A multi-centre phase 3 study comparing efficacy and safety of Bemfola ${ }^{\circledR}$ versus Gonal-f in women undergoing ovarian stimulation for IVF. 2015, Reproductive BioMedicine Online.

7. Ministero della Salute. 2014 йил. Available from: http://www.salute. gov.it/. Accessed March 1, 2016.

8. MSSSI. Registro de Altas de los Hospitales Generales del Sistema Nacional de Salud. [Online] CMBD. Norma Estatal., 2013. Available from: https://www.msssi.gob.es/estadEstudios/estadisticas/cmbdhome. htm. Accessed March 1, 2016.

9. Gazzetta Ufficiale. Elenco Atti dell'emittente AGENZIA ITALIANA DEL FARMACO. [Online]. Available from: http://www.gazzett aufficiale.it/ Accessed March 1, 2016.

10. Gisbert R, Brosa M. eSalud Sanitary costs database. [Online] Barcelona: Oblikue Consulting; 2016. http://www.oblikue.com/en/index.htm. Accessed March 1, 2016.

11. Young KE, Rémuzat C, Urbinati D, Toumi M. Boosting Biosimilars Uptake in European Countries. ISPOR 17th Annual European Congress, 8-12 November, 2014, Amsterdam, The Netherlands; PHP23.

12. Centro Studi e Ricerche sulle Biotecnologie Sanitarie e Settore Biotech. La diffusione dei farmaci biotecnologici, la loro costo-efficacia e i trend legati all'associazione farmaco-test per biomarker; 2014.

13. All Wales Medicines Strategy Group; 2014. http://www.awmsg.org/ awmsgonline/app/appraisalinfo/2088. Accessed March 1, 2016.

14. Scottish Medicines Consortium. Benfola - summary of comparative health economic evidence. 2015.

15. Ocal P, Sahmay S, Cetin M, Irez T, Guralp O, Cepni I. Serum anti-Mullerian hormone and antral follicle count as predictive markers of OHSS in ART cycles. J Assist Reprod Genet. 2011;28(12):1197-1203.

16. Lee TH, Liu CH, Huang CC, et al. Serum anti-Mullerian hormone and estradiol levels as predictors of ovarian hyperstimulation syndrome in assisted reproduction technology cycles. Hum Reprod. 2008;23(1): $160-167$.
International Journal of Women's Health

\section{Publish your work in this journal}

The International Journal of Women's Health is an international, peerreviewed open-access journal publishing original research, reports, editorials, reviews and commentaries on all aspects of women's healthcare including gynecology, obstetrics, and breast cancer. The manuscript management system is completely online and includes

\section{Dovepress}

a very quick and fair peer-review system, which is all easy to use. Visit http://www.dovepress.com/testimonials.php to read real quotes from published authors. 